\title{
SYNTHESIS OF NEW 1,2,4-TRIAZOLE DERIVATIVES OF NALIDIXIC ACID AS POTENTIAL ANTIBACTERIAL AND ANTIFUNGAL AGENTS
}

\author{
Samia G. Abdelmoty ${ }^{*}$ and Helal F. Heta ${ }^{2}$
}

${ }^{1}$ Department of Pharmaceutical Organic Chemistry, Faculty of Pharmacy, Assiut University, Assiut 71526, Egypt,

${ }^{2}$ Department of Microbiology and Immunology, Faculty of Medicine, Assiut University, Assiut 71526, Egypt

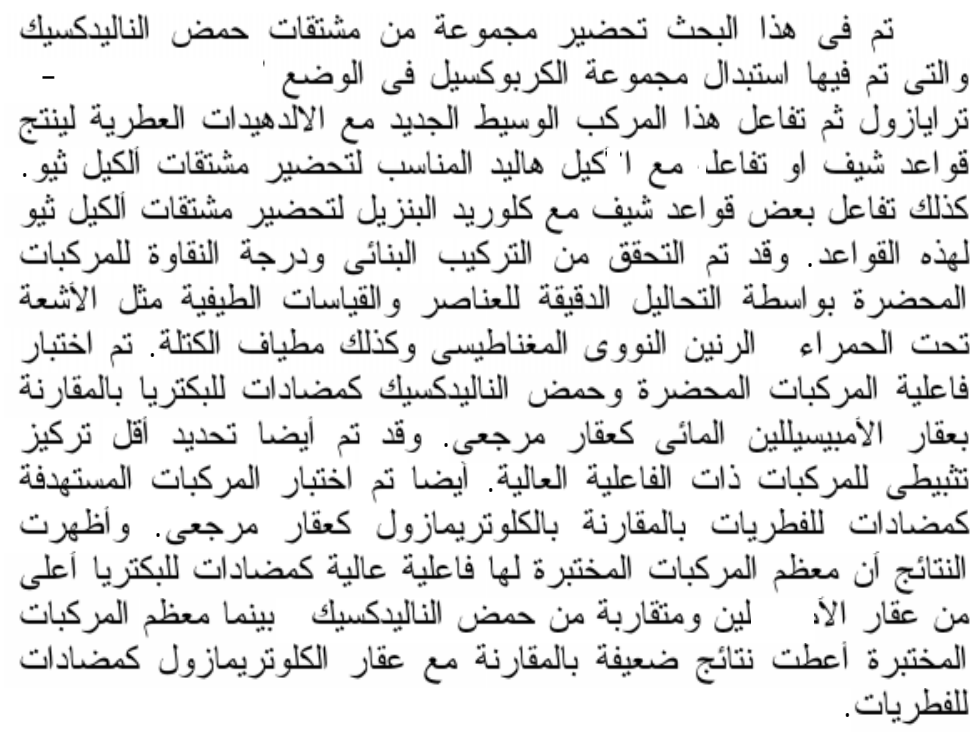

Triazole and triazole fused heterocyclic ring systems possess diverse applications in the fields of medicine, agriculture and industry. A new series of nalidixic acid derivatives having 1,2,4triazole moiety at position 3 were synthesised to achieve enhanced biological activity and wide spectrum of activity. Nalidixic acid was first converted into its methyl ester which upon hydrazinolysis afforded nalidixic acid hydrazide. Condensation of the hydrazide with $\mathrm{CS}_{2} / \mathrm{KOH}$ furnished the potassium dithiocarbazate salt, which cyclized to the 3-[4-amino-5-thioxo-4,5-dihydro-1H-1,2,4-triazol3-yl]-1-ethyl-7-methyl-1H-[1,8]naphthyridin-4-one, (4), on

Received in 8/2/2009, Received in revised form in 28/3/2009 \& Accepted in 30/3/2009

${ }^{*}$ Corresponding author e-mail address: abdelmoty99@yahoo.com 
refluxing with hydrazine hydrate. Condensation of the key intermediate 4 with aryl aldehydes afforded Schiff's bases 5a-f, while its reaction with alkyl or aralkyl halides gave compounds 6a-e. Furthermore, compounds 5a,e were reacted with benzyl chloride to afford $7 a, b$. The chemical structure of the target compounds was confirmed by IR, ${ }^{l} H-N M R, F A B-M S$, EI-MS spectra and elemental analyses. The title compounds and the starting Nalidixic acid; were tested for their in-vitro antibacterial and antifungal activities. Most of the tested compounds showed comparable antibacterial activity with those of Nalidixic acid and higher activity than ampicillin. The tested compounds and Nalidixic acid showed non or moderate antifungal activity in comparison to clotrimazole as a reference drug.

\section{INTRODUCTION}

The 1,8-naphthyridine nucleus (the main skeleton of nalidixic acid) is one of the frequently found heterocyclic moiety among different biologically active compounds ${ }^{1-7}$. Nalidixic acid (trade name NegGram) is the basis for quinoline antibiotics which is effective against both Grampositive and Gram-negative bacteria. It was introduced for the treatment of urinary tract infections caused, for example, by Escherichia coli, Proteus, Shigella, Enterobacter, and Klebsiella $^{1 \& 2}$. It failed to achieve adequate concentrations in the plasma or tissues for the treatment of systemic infections following oral or potential administration but got concentrated in the urine, where they could be effective for eradicating urinary tract infections ${ }^{1}$. Structure activity relationship (SAR) of compounds based on nalidixic acid, have led to a large group of synthetic antibacterial agents collectively known as the quinolones ${ }^{2,8 \& 9}$. Resistance was found to emerge rapidly, even while on therapy due to the increasing of resistance of many infections by Gram negative and Gram positive bacteria. The emergence of multidrug resistance is a problem of ever increasing significance $^{10}$. Organisms including methicillin-resistant Staphylococcus aureus (MRSA) and Staphylococcus epidermids (MRSE), vancomycinresitant enterococci (VRE), penicillin and cephalosporin-resistant streptococci are continuously challenging chemists, physicians and patients. Consequently, the search of new chemotherapeutic agents constitutes real challenge facing microbiologists, pharmacologists as well as medicinal chemists.

Currently, 1,2,4-triazole nucleus has been incorporated in a wide variety of pharmacologically active compounds. It represents one of the most biologically active classes of compounds, possessing a wide 
spectrum of activities. Also, it has been observed that 1,2,4-triazole moiety has a great versatility in fusing to various ring systems and the $\mathrm{N}$ bridged heterocycles derived from them are associated with diverse pharmacological activity such as antibacterial, antifungal, hypoglycemic, antihypertensive and analgesic properties $^{11-13}$.

In the present work, these encouraging facts mentioned above about biological activity associated with both 1,2,4-triazoles and nalidixic acid moieties ${ }^{14}$ prompted us to synthesize some nalidixic acid derivatives carrying the biodynamic heterocyclic system (1,2,4-triazole) at position-3 with an objective to obtain biheterocycles of enhanced biological activity. The compounds so obtained were screened for their in-vitro activity against Gram-positive, Gramnegative bacteria and different phytopathogenic fungi species.

\section{EXPERIMENTAL}

\section{Chemistry}

\section{Materials and equipments}

Melting points were determined on an electrothermal melting point apparatus (Stuart Scientific, model SMP1, UK), and were uncorrected. Precoated silica gel plates (kieselgel $0.2 \mathrm{~mm}, 60 \mathrm{G} \mathrm{F}_{254}$, Merck) were used for thin layer chromatography. A developing solvent system of chloroform/methanol $(9: 1)$ was used and the spots were detected by ultraviolet lamp (Spectroline, model CM-10, U.S.A.).
IR spectra ( $\mathrm{KBr}$ discs) were recorded on a Shimadzu spectrophotometer (IR-470), Faculty of Pharmacy, Assiut University, Assiut. ${ }^{1} \mathrm{H}-\mathrm{NMR}$ Spectra were performed on a Varian EM-360 $60 \mathrm{MHz}$ NMR spectrometer, Faculty of Pharmacy, Assiut University, Assiut. Chemical shifts are expressed in $\delta$-value (ppm) relative to TMS as an internal standard, using DMSO-d $\mathrm{d}_{6}$ or $\mathrm{CDCl}_{3}$ as solvents and deuterium oxide was used for the detection of the exchangeable protons. Mass spectra were recorded on Jeol JMS mass spectrometer, at Assiut University Central Lab., Assiut University, Assiut. Elemental analyses were performed on a Perkin-Elmer 240 elemental analyzer at the unit of microanalysis, Faculty of Science, Assiut University, Assiut, Egypt.

Nalidixic acid acid was offered by El-Nasr chemical industry co. (Cairo/ Egypt). Other materials used for the synthesis of the different intermediates required in this work are commercially available from Aldrich and include alkyl, aralkyl halides and (un)substituted benzaldehydes. All other chemicals and solvents are of the reagent grade.

The following starting materials were prepared according to reported methods: Methyl 1-ethyl-1,4-dihydro7-methyl-4-oxo-1,8-naphthyridine-3carboxylate $(\mathbf{2})^{15}$ and 1-ethyl-1,4dihydro-7-methyl-4-oxo-1,8-naphthyridine-3-carboxylic acid hydrazide $(3)^{16}$. 
3-[4-Amino-5-thioxo-4,5-dihydro1H-1,2,4-triazol-3-yl]-1-ethyl-7methyl-1H-1,8-naphthyridin-4one (4)

To a stirred ice-cooled solution of 1-ethyl-1,4-dihydro-7-methyl-4-oxo-

1,8-naphthyridine-3-carboxylic acid hydrazide (3) (2.46 g, $0.01 \mathrm{~mol})$ and potassium hydroxide $(0.84 \mathrm{~g}, 0.015$ $\mathrm{mol})$ in absolute ethanol $(60 \mathrm{~mL})$, carbon disulfide $(1 \mathrm{~mL}, 0.015 \mathrm{~mol})$ was added. The reaction mixture was stirred at room temperature for $20 \mathrm{~h}$ whereupon a yellow precipitate of the corresponding potassium dithiocarbazate was separated. Dry ether $(50 \mathrm{~mL})$ was then added to complete the precipitation. The product was filtered off, washed several times with dry ether and dried. The salt was then suspended in hydrazine hydrate $97 \%$ $(1 \mathrm{~mL}, 0.02 \mathrm{~mol})$ with stirring and heated under reflux for $2 \mathrm{hrs}$. The reaction mixture was cooled, diluted with ice-cold water $(50 \mathrm{~mL})$ and neutralized with $10 \%$ hydrochloric $\operatorname{acid}^{11}$. The precipitate obtained was filtered, washed thoroughly with cold water and crystallized from dimethylformamide / water affording $2.4 \mathrm{~g}(80 \%)$ of a white solid: m.p. $258-260^{\circ} \mathrm{C}$.

IR, $v^{-} \mathrm{cm}^{-1}(\mathrm{KBr}): 3455,3250$ $\left(\mathrm{NH}_{2}, \mathrm{NH}\right), 3130$ (Ar. $\left.\mathrm{CH}\right), 2920$ (aliph. CH), $1614 \quad(\mathrm{C}=\mathrm{O}), \quad 1558$ $(\mathrm{C}=\mathrm{N}), 1527(\mathrm{~N}-\mathrm{H}), 1251(\mathrm{C}=\mathrm{S})$.

${ }^{1} \mathrm{H}-\mathrm{NMR}, \delta$ ppm (DMSO-d $\left.{ }_{6}\right): 1.50$ (t, 3H, $\left.\mathrm{CH}_{2}-\mathrm{CH}_{3}\right) ; 2.80$ (s, 3H, C7$\left.\mathrm{CH}_{3}\right) ; 4.80$ (q, $\left.2 \mathrm{H}, \mathrm{CH}_{2}-\mathrm{CH}_{3}\right) ; 6.13$ (br. s, 2H, $\underline{\mathrm{N}}_{2}$ ); 7.92 (d, 1H, C6- $\underline{\mathrm{H}}$ ); $9.13(\mathrm{~d}, 1 \mathrm{H}, \mathrm{C} 5-\underline{\mathrm{H}}) ; 9.30$ (s, 1H, C2-
$\underline{\mathrm{H}}$; 15.16 (br. s, 1H, NH). Anal. Calcd for $\mathrm{C}_{13} \mathrm{H}_{14} \mathrm{~N}_{6} \mathrm{OS} \quad(\mathrm{M} \quad \mathrm{W}$ 302.09): \%C 51.64, \% $4.67, \% \mathrm{~N}$ 27.80, \%S 10.61. Found: \%C 51.59, $\% \mathrm{H} 4.24, \% \mathrm{~N} 27.62, \% \mathrm{~S} 10.91$.

\section{Synthesis of 3-\{[4-(un)substituted benzylidene-amino]-5-thioxo-4,5- dihydro-1 $H$ - $(1,2,4)$ triazol-3-yl\}-1- ethyl-7-methyl-1H-[1,8]naphthy- ridin-4-one derivatives (5a-f)}

To a suspension of 3-[4-amino-5thioxo-4,5-dihydro- $1 H$ - $(1,2,4)$ triazole-3-yl]-1-ethyl-7-methyl- $1 \mathrm{H}$ 1,8-naphthyridin-4-one, compound 4 $(1.51 \mathrm{~g}, 0.005 \mathrm{~mol})$ in absolute ethanol $(25 \mathrm{~mL})$, the appropriate (un)substituted aryl aldehyde (0.005 mol) was added, treated with concentrated $\mathrm{HCl}(0.5 \mathrm{~mL})$ and refluxed for $20 \mathrm{hrs}^{17}$. The reaction mixture was cooled to the ambient temperature and the formed precipitate was filtered and recrystallized from ethanol to yield the compounds (5a-f). The physicochemical data are listed in Table I.

3-\{[4-Benzylidene-amino]-5-thioxo4,5-dihydro-1H-(1,2,4)triazol-3-yl\}1-ethyl-7-methyl-1H-[1,8]naphthyridin-4-one (5a)

${ }^{1} \mathrm{H}-\mathrm{NMR}$ (DMSO- $\mathrm{d}_{6}, \delta$ ppm): 1.50 (t, $\left.3 \mathrm{H}, \mathrm{N}-\mathrm{CH}_{2} \mathrm{CH}_{3}\right) ; 2.86$ (s, 3H, C7$\left.\mathrm{C}_{3}\right) ; 4.81$ (q, $\left.2 \mathrm{H}, \quad \mathrm{N}-\underline{\mathrm{C}}_{2}-\mathrm{CH}_{3}\right)$; 7.76-8.46 (m, 6H, $\mathrm{C}_{6} \underline{\mathrm{H}}_{5}$ and $\mathrm{C} 6-\underline{\mathrm{H}}$ ); $9.00(\mathrm{~d}, 1 \mathrm{H}, \mathrm{C} 5-\underline{\mathrm{H}}) ; 9.30(\mathrm{~s}, 1 \mathrm{H}, \mathrm{C} 2-$ $\underline{\mathrm{H}}) ; 10.86(\mathrm{~s}, 1 \mathrm{H}, \mathrm{N}=\mathrm{C} \underline{\mathrm{H}}) ; 15.53$ (br. $\mathrm{s}, 1 \mathrm{H}, \mathrm{N} \underline{\mathrm{H}}$ exchanged by $\mathrm{D}_{2} \mathrm{O}$ ). 
Table I: Physicochemical data of compounds (5 a-f).

\begin{tabular}{|c|c|c|c|c|c|c|c|c|}
\hline \multirow{2}{*}{$\begin{array}{l}\text { Comp. } \\
\text { No. }\end{array}$} & \multirow{2}{*}{$\mathrm{R}$} & \multirow{2}{*}{$\begin{array}{l}\text { M. formula } \\
\text { (M.W.) }\end{array}$} & \multirow{2}{*}{$\begin{array}{l}\text { m.p. } \\
\left({ }^{\circ} \mathrm{C}\right)\end{array}$} & \multirow{2}{*}{$\begin{array}{l}\text { Yield } \\
(\%)\end{array}$} & \multicolumn{4}{|c|}{$\begin{array}{l}\text { Microanalyses } \\
\text { Calcd. / Found }\end{array}$} \\
\hline & & & & & $\mathrm{C} \%$ & $\mathrm{H} \%$ & $\mathrm{~N} \%$ & $\mathrm{~S} \%$ \\
\hline $5 a$ & $\mathrm{H}$ & $\begin{array}{c}\mathrm{C}_{20} \mathrm{H}_{18} \mathrm{~N}_{6} \mathrm{OS} \\
(390.13) \\
\end{array}$ & $300-302$ & 75 & $\begin{array}{l}61.52 \\
62.38 \\
\end{array}$ & $\begin{array}{l}4.65 \\
4.64 \\
\end{array}$ & $\begin{array}{l}21.52 \\
21.41 \\
\end{array}$ & $\begin{array}{l}8.21 \\
8.40 \\
\end{array}$ \\
\hline $5 b$ & $p-\mathrm{Br}$ & $\begin{array}{c}\mathrm{C}_{20} \mathrm{H}_{17} \mathrm{BrN}_{6} \mathrm{OS} \\
(468.04)\end{array}$ & $270-2$ & 73 & $\begin{array}{l}51.18 \\
51.55 \\
\end{array}$ & $\begin{array}{l}3.65 \\
3.92 \\
\end{array}$ & $\begin{array}{l}17.91 \\
17.89 \\
\end{array}$ & $\begin{array}{l}6.83 \\
6.82 \\
\end{array}$ \\
\hline $5 c$ & $p-\mathrm{Cl}$ & $\begin{array}{c}\mathrm{C}_{20} \mathrm{H}_{17} \mathrm{ClN}_{6} \mathrm{OS} \\
(424.09) \\
\end{array}$ & $275-7$ & 68 & $\begin{array}{l}56.53 \\
56.29 \\
\end{array}$ & $\begin{array}{l}4.03 \\
4.03 \\
\end{array}$ & $\begin{array}{l}19.78 \\
20.37 \\
\end{array}$ & $\begin{array}{l}7.55 \\
7.70 \\
\end{array}$ \\
\hline $5 d$ & $o-\mathrm{OCH}_{3}$ & $\begin{array}{c}\mathrm{C}_{21} \mathrm{H}_{20} \mathrm{~N}_{6} \mathrm{O}_{2} \mathrm{~S} \\
(420.14) \\
\end{array}$ & $255-7$ & 71 & $\begin{array}{l}59.98 \\
59.30 \\
\end{array}$ & $\begin{array}{l}4.79 \\
5.28 \\
\end{array}$ & $\begin{array}{l}19.91 \\
20.12 \\
\end{array}$ & $\begin{array}{l}7.63 \\
8.12 \\
\end{array}$ \\
\hline $5 \mathrm{e}^{*}$ & $p-\mathrm{OCH}_{3}$ & $\begin{array}{c}\mathrm{C}_{21} \mathrm{H}_{20} \mathrm{~N}_{6} \mathrm{O}_{2} \mathrm{~S} \\
1 / 2 \mathrm{H}_{2} \mathrm{O} \\
(429.14) \\
\end{array}$ & 298-300 & 70 & $\begin{array}{l}58.72 \\
58.20\end{array}$ & $\begin{array}{l}4.69 \\
4.34\end{array}$ & $\begin{array}{l}19.57 \\
19.96\end{array}$ & $\begin{array}{l}7.45 \\
7.63\end{array}$ \\
\hline $5 f$ & $p-\mathrm{CH}_{3}$ & $\begin{array}{c}\mathrm{C}_{21} \mathrm{H}_{20} \mathrm{~N}_{6} \mathrm{OS} \\
(404.49) \\
\end{array}$ & $230-2$ & 60 & $\begin{array}{l}62.36 \\
61.36 \\
\end{array}$ & $\begin{array}{l}4.98 \\
5.21 \\
\end{array}$ & $\begin{array}{l}20.78 \\
20.78 \\
\end{array}$ & $\begin{array}{l}7.93 \\
8.29 \\
\end{array}$ \\
\hline
\end{tabular}

* It was further confirmed by FAB MS m/z, (\%): $421.18[\mathrm{M}+1]^{+}(2.2 \%), 287.93$ (100\%) 214.01 (36.8\%), 185.95 (46.4\%), 92.94 (49.4\%) and 78.95 (86.2)

3-\{[4-(p-Bromo-benzylidene)amino]-5-thioxo-4,5-dihydro- $1 H$ $(1,2,4)$ triazol-3-yl\}-1-ethyl-7methyl-1H-[1,8]naphthyridin-4one $(5 \mathbf{b})$

${ }^{1} \mathrm{H}-\mathrm{NMR}$ (DMSO-d $\mathrm{d}_{6}, \delta \mathrm{ppm}$ ): 1.53 (t, $3 \mathrm{H}, \mathrm{N}-\mathrm{CH}_{2} \mathrm{C}_{3}$ ); 2.86 (s, 3H, C7$\left.\mathrm{C}_{3}\right) ; 4.90\left(\mathrm{q}, 2 \mathrm{H}, \mathrm{N}-\mathrm{C}_{2}-\mathrm{CH}_{3}\right) ; 8.03$ (d, $1 \mathrm{H}, \mathrm{C} 6-\underline{\mathrm{H}}) ; 8.35$ (s, 4H, $\left.\mathrm{C}_{6} \underline{\mathrm{H}}_{4}\right)$; $9.20(\mathrm{~d}, 1 \mathrm{H}, \mathrm{C} 5-\underline{\mathrm{H}}) ; 9.46(\mathrm{~s}, 1 \mathrm{H}, \mathrm{C} 2-$ $\underline{\mathrm{H}}) ; 10.80(\mathrm{~s}, 1 \mathrm{H}, \mathrm{N}=\mathrm{C} \underline{\mathrm{H}}) ; 15.47$ (br. $\mathrm{s}, 1 \mathrm{H}, \mathrm{NH}$ exchanged by $\left.\mathrm{D}_{2} \mathrm{O}\right)$.

\section{3-\{[4-(p-Chloro-benzylidene)-} amino]-5-thioxo-4,5-dihydro- $1 H$ $(1,2,4)$ triazol-3-yl\}-1-ethyl-7methyl-1H-[1,8]naphthyridin-4one $(5 c)$

${ }^{1} \mathrm{H}-\mathrm{NMR}$ (DMSO-d ${ }_{6}, \delta \mathrm{ppm}$ ): 1.54 (t, 3H, N-CH ${ }_{2} \mathrm{CH}_{3}$ ); 2.83 (s, 3H, C7$\left.\mathrm{C}_{3}\right) ; 4.88$ (q, $\left.2 \mathrm{H}, \quad \mathrm{N}-\mathrm{C}_{2}-\mathrm{CH}_{3}\right)$; 7.80-8.56 (m, 5H, $\mathrm{C}_{6} \mathrm{H}_{4}$ and $\left.\mathrm{C} 6-\mathrm{H}\right)$; $9.0(\mathrm{~d}, 1 \mathrm{H}, \mathrm{C} 5-\underline{\mathrm{H}}) ; 9.30(\mathrm{~s}, 1 \mathrm{H}, \mathrm{C} 2-\underline{\mathrm{H}})$; $10.93(\mathrm{~s}, 1 \mathrm{H}, \mathrm{N}=\mathrm{C} \underline{\mathrm{H}}) ; 15.56$ (br. s, $1 \mathrm{H}$, $\mathrm{NH}$ exchanged by $\mathrm{D}_{2} \mathrm{O}$ ). 
3-\{[4-(o-Methoxy-benzylidene)amino]-5-thioxo-4,5-dihydro-1H$(1,2,4)$ triazol-3-yl\}-1-ethyl-7methyl-1H-[1,8]naphthyridin-4one (5d)

${ }^{1} \mathrm{H}-\mathrm{NMR}$ (DMSO-d $\left.\mathrm{d}_{6}, \delta \mathrm{ppm}\right): 1.50$ (t, 3H,N-CH$\left.{ }_{2} \mathrm{CH}_{3}\right) ; 2.80$ (s, 3H, C7$\left.\mathrm{C}_{\underline{3}}\right) ; 4.16$ (s, $\left.3 \overline{\mathrm{H}}, \mathrm{OC}_{\underline{3}}\right) ; 4.80$ (q, $\left.2 \mathrm{H}, \mathrm{N}-\mathrm{CH}_{2}-\mathrm{CH}_{3}\right) ; 7.20-8.46(\mathrm{~m}, 5 \mathrm{H}$, $\mathrm{C}_{6} \underline{\mathrm{H}}_{4}$ and $\left.\mathrm{C} 6-\underline{\mathrm{H}}\right) ; 8.93$ (d, 1H, C5- $\underline{\mathrm{H}}$ ); $9.25(\mathrm{~s}, 1 \mathrm{H}, \mathrm{C} 2-\underline{\mathrm{H}}) ; 11.2(\mathrm{~s}, 1 \mathrm{H}$, $\mathrm{N}=\mathrm{C} \underline{\mathrm{H}}$ ); 15.53 (br. s, $1 \mathrm{H}, \quad \mathrm{NH}$ exchanged by $\mathrm{D}_{2} \mathrm{O}$ ).

3-\{[4-(p-Methoxy-benzylidene)amino]-5-thioxo-4,5-dihydro- $1 H$ $(1,2,4)$ triazol-3-yl\}-1-ethyl-7methyl-1H-[1,8]naphthyridin-4one (5e)

${ }^{1} \mathrm{H}-\mathrm{NMR}$ (DMSO-d 6 , $\delta$ ppm): 1.50 (t, 3H, N-CH $\left.\mathrm{CH}_{3}\right) ; 2.83$ (s, 3H, C7$\left.\mathrm{CH}_{3}\right) ; 4.13$ (s, 3H, OCH $\left.\underline{\mathrm{H}}_{3}\right) ; 4.86$ (q, $\left.2 \mathrm{H}, \mathrm{N}-\mathrm{CH}_{2}-\mathrm{CH}_{3}\right) ; 7.56$ (d, 1H, C6$\underline{\mathrm{H}}) ; 7.93$ and $8.37\left(\mathrm{dd}, 4 \mathrm{H}, \mathrm{C}_{6} \mathrm{H}_{4}\right) ; 9.10$ (d, 1H, C5- $\underline{\mathrm{H}}) ; 9.35(\mathrm{~s}, 1 \mathrm{H}, \mathrm{C} 2-\underline{\mathrm{H}})$; 10.56 (s, $1 \mathrm{H}, \mathrm{N}=\mathrm{CH}$ ); 15.37 (br. s, $1 \mathrm{H}, \mathrm{NH}$ exchanged by $\left.\mathrm{D}_{2} \mathrm{O}\right)$.

FAB MS $m / z,(\%): 421.18[\mathrm{M}+1]^{+}$ $(2.2 \%), \quad 287.93 \quad(100 \%), \quad 214.01$ $(36.8 \%), \quad 185.95 \quad(46.4 \%), \quad 92.94$ $(49.4 \%)$ and 78.95 (86.2).

\section{3-\{[4-(p-Methyl-benzylidene)-} amino]-5-thioxo-4,5-dihydro-1H$(1,2,4)$ triazol-3-yl\}-1-ethyl-7methyl-1H-[1,8]naphthyridin-4one (5f)

${ }^{1} \mathrm{H}-\mathrm{NMR}$ (DMSO-d $\left.\mathrm{d}_{6}, \delta \mathrm{ppm}\right): 1.56$ (t, 3H, N- $\left.\mathrm{CH}_{2} \mathrm{CH}_{3}\right) ; 2.55$ (s, 3H, p$\mathrm{CH}_{3}-\mathrm{ph}$ ); 2.86 (s, $3 \mathrm{H}, \mathrm{C} 7-\mathrm{CH}_{3}$ ); 4.86 (q, $\left.2 \mathrm{H}, \mathrm{N}-\mathrm{CH}_{2}-\mathrm{CH}_{3}\right) ; 7.53-8.43$ (m, $5 \mathrm{H}, \mathrm{C}_{6} \underline{\mathrm{H}}_{4}$ and $\left.\mathrm{C} 6-\mathrm{H}\right) ; 8.93$ (s, 1H, C2$\underline{\mathrm{H}}) ; 9.25(\mathrm{~d}, 1 \mathrm{H}, \mathrm{C} 5-\underline{\mathrm{H}}) ; 10.85(\mathrm{~s}, 1 \mathrm{H}$,
$\mathrm{N}=\mathrm{CH}$ ); 12.50 (br. s, 1H, $\mathrm{NH}$ exchanged by $\mathrm{D}_{2} \mathrm{O}$ ).

Synthesis of 3-[4-amino-5-alkylthio$4 H-(1,2,4)$ triazol-3-yl]-1-ethyl-7methyl-1H-[1,8]naphthyridin-4one derivatives (6a-e)

The appropriate alkyl or aralkyl halide $(0.0024 \mathrm{~mol})$ in absolute ethanol $(30 \mathrm{~mL})$ was added to 3[4-amino-5-thioxo-4,5-dihydro- $1 \mathrm{H}$ $(1,2,4)$-triazol-3-yl]-1-ethyl-7-methyl$1 \mathrm{H}$-1,8-naphthyridin-4-one,compound $4(0.6 \mathrm{~g}, 0.002 \mathrm{~mol})$ dissolved in a least amount of $10 \% \mathrm{NaOH}^{18}$. The reaction mixture was refluxed for 15 hrs, and then concentrated under reduced pressure. The residue obtained was filtered washed with water and crystallized from ethyl acetate to yield compounds (6a-e). The physicochemical data are listed in Table II.

\section{3-[4-Amino-5-methylthio-4H- $(1,2,4)$ triazol-3-yl]-1-ethyl-7- methyl-1H-[1,8]naphthyridin-4- one (6a)}

IR, $v^{-} \mathrm{cm}^{-1}$ (KBr): 3400 and 3260 $\left(\mathrm{NH}_{2}\right), 3010$ (Ar. CH), 2950 (aliph. $\mathrm{CH}), 1619(\mathrm{C}=\mathrm{O}), 1566(\mathrm{NH}, \mathrm{C}=\mathrm{N})$.

${ }^{1} \mathrm{H}-\mathrm{NMR} \quad\left(\mathrm{CDCl}_{3}+\mathrm{DMSO}_{6}\right.$ (4:1), $\delta \mathrm{ppm}): 1.65$ (t, $3 \mathrm{H}, \mathrm{CH}_{2}-\mathrm{CH}_{3}$ ); $2.93\left(\mathrm{~s}, 3 \mathrm{H}, \mathrm{C} 7-\mathrm{CH}_{3}\right) ; 3.00(\mathrm{~s}, 3 \mathrm{H}, \mathrm{S}$ $\mathrm{CH} 3$ ); 5.00 (q, 2H, N-C $\underline{\mathrm{H}}_{2}-\mathrm{CH}_{3}$ ); 6.40 (br. s, $2 \mathrm{H}, \mathrm{NH}_{2}$, exchanged by $\mathrm{D}_{2} \mathrm{O}$ ); $8.00 \mathrm{~d}, 1 \mathrm{H}, \mathrm{C} 5-\mathrm{H}) ; 9.26$ (s, 1H, C2$\mathrm{H}) ; 9.36$ (d, 1H, C6-H).

FAB-MS, $m / z, \%: 317.29[\mathrm{M}+1]^{+}$, $(95 \%), \quad 302.27 \quad(22.7 \%), \quad 214.24$ (17.6\%), $\quad 185.26 \quad(50 \%), \quad 93.10$ $(100 \%)$. 
Table II: Physicochemical data of compounds (6a-e).<smiles>CCCc1nnc(-c2cn(CC)c3nc(C)ccc3c2=O)n1N</smiles>

\begin{tabular}{|c|c|c|c|c|c|c|c|c|}
\hline \multirow{2}{*}{$\begin{array}{l}\text { Comp. } \\
\text { No. }\end{array}$} & \multirow{2}{*}{$\mathrm{R}_{1}$} & \multirow{2}{*}{$\begin{array}{l}\text { M. Formula* } \\
\text { (M.W.) }\end{array}$} & \multirow{2}{*}{$\begin{array}{l}\text { m.p. } \\
\left({ }^{\circ} \mathrm{C}\right)\end{array}$} & \multirow{2}{*}{$\begin{array}{l}\text { Yield } \\
(\%)\end{array}$} & \multicolumn{4}{|c|}{$\begin{array}{l}\text { Microanalyses } \\
\text { Calcd/ Found }\end{array}$} \\
\hline & & & & & $\mathrm{C} \%$ & $\mathrm{H} \%$ & $\mathrm{~N} \%$ & $\mathrm{~S} \%$ \\
\hline $6 a$ & $\mathrm{CH}_{3}$ & $\begin{array}{c}\mathrm{C}_{14} \mathrm{H}_{16} \mathrm{~N}_{6} \mathrm{OS} \\
1.5 \mathrm{H}_{2} \mathrm{O} \\
(343.12)\end{array}$ & $\begin{array}{l}188- \\
190\end{array}$ & 65 & $\begin{array}{l}48.96 \\
48.22\end{array}$ & $\begin{array}{l}4.70 \\
4.55\end{array}$ & -- & $\begin{array}{l}9.32 \\
8.86\end{array}$ \\
\hline $6 \mathbf{b}$ & $\mathrm{C}_{2} \mathrm{H}_{5}$ & $\begin{array}{c}\mathrm{C}_{15} \mathrm{H}_{18} \mathrm{~N}_{6} \mathrm{OS} \\
1.5 \mathrm{H}_{2} \mathrm{O} \\
(357.12)\end{array}$ & $\begin{array}{l}170- \\
172\end{array}$ & 67 & $\begin{array}{l}50.40 \\
49.87\end{array}$ & $\begin{array}{l}5.08 \\
6.09\end{array}$ & -- & $\begin{array}{l}8.95 \\
8.58\end{array}$ \\
\hline $6 c$ & $\mathrm{n}-\mathrm{C}_{3} \mathrm{H}_{7}$ & $\begin{array}{c}\mathrm{C}_{16} \mathrm{H}_{20} \mathrm{~N}_{6} \mathrm{OS} \\
1.5 \mathrm{H}_{2} \mathrm{O} \\
(371.16)\end{array}$ & $\begin{array}{l}177- \\
179\end{array}$ & 65 & $\begin{array}{l}51.73 \\
51.46\end{array}$ & $\begin{array}{l}5.43 \\
5.98\end{array}$ & $\begin{array}{l}22.63 \\
22.81\end{array}$ & $\begin{array}{l}8.61 \\
9.57\end{array}$ \\
\hline 6d & $\mathrm{CH}_{2} \mathrm{CH}=\mathrm{CH}_{2}$ & $\begin{array}{c}\mathrm{C}_{16} \mathrm{H}_{18} \mathrm{~N}_{6} \mathrm{OS} \\
0.5 \mathrm{H}_{2} \mathrm{O} \\
(351.14)\end{array}$ & $\begin{array}{l}167- \\
169\end{array}$ & 62 & $\begin{array}{l}54.68 \\
54.04\end{array}$ & $\begin{array}{l}5.17 \\
5.28\end{array}$ & -- & $\begin{array}{l}9.10 \\
8.54\end{array}$ \\
\hline $6 e$ & $\mathrm{CH}_{2} \mathrm{C}_{6} \mathrm{H}_{5}$ & $\begin{array}{c}\mathrm{C}_{20} \mathrm{H}_{20} \mathrm{~N}_{6} \mathrm{OS} \\
1.5 \mathrm{H}_{2} \mathrm{O} \\
(419.15)\end{array}$ & $\begin{array}{l}166- \\
168\end{array}$ & 79 & $\begin{array}{l}57.26 \\
57.56\end{array}$ & $\begin{array}{l}4.81 \\
5.39\end{array}$ & $\begin{array}{l}20.08 \\
19.91\end{array}$ & $\begin{array}{l}7.63 \\
7.47\end{array}$ \\
\hline
\end{tabular}

* All compounds are further confirmed by EI and FAB MS (see the exp. part).

3-[4-Amino-5-ethylthio-4H-(1,2,4)triazol-3-yl]-1-ethyl-7-methyl-1H$[1,8]$ naphthyridin-4-one $(6 \mathrm{~b})$

${ }^{1} \mathrm{H}-\mathrm{NMR}\left(\mathrm{CDCl}_{3}, \delta \mathrm{ppm}\right): 2.16-$ $1.36\left(\mathrm{~m}, 6 \mathrm{H}, 2 \mathrm{CH}_{2}-\mathrm{C}_{3}\right) ; 2.96(\mathrm{~s}, 3 \mathrm{H}$, $\left.\mathrm{C} 7-\mathrm{CH}_{3}\right) ; 3.53$ (q, $\left.2 \mathrm{H}, \mathrm{S}-\mathrm{CH}_{2}-\mathrm{CH}_{3}\right)$; $5.00\left(\mathrm{q}, 2 \mathrm{H}, \mathrm{N}-\mathrm{C}_{2}-\mathrm{CH}_{3}\right) 6.28$ (br. s, $2 \mathrm{H}, \mathrm{NH}_{2}$, exchanged by $\left.\mathrm{D}_{2} \mathrm{O}\right) ; 7.93$ $(\mathrm{d}, 1 \mathrm{H}, \mathrm{C} 5-\mathrm{H}) ; 9.20(\mathrm{~s}, 1 \mathrm{H}, \mathrm{C} 2-\mathrm{H})$; 9.36(d, 1H, C6-H).
EI-MS, $m / z, \%: 330\left(\mathrm{M}^{+}, 90 \%\right)$ $314\left(\mathrm{M}^{+}-\mathrm{NH}_{2}, 97 \%\right), 214(67 \%)$, $186(100 \%)$.

3-[4-Amino-5-n-propylthio-4H-(1,2, 4)triazol-3-yl]-1-ethyl-7-methyl-1H[1,8]naphthyridin-4-one (6c)

${ }^{1} \mathrm{H}-\mathrm{NMR}\left(\mathrm{CDCl}_{3}, \delta \mathrm{ppm}\right): 1.10(\mathrm{t}$, $\left.3 \mathrm{H}, \quad \mathrm{CH}_{2}-\mathrm{CH}_{2}-\mathrm{CH}_{3}\right) ; 1.46-2.26(\mathrm{~m}$, $5 \mathrm{H}, \mathrm{CH}_{2}-\underline{\mathrm{C}}_{3}$ and $\left.\mathrm{CH}_{2}-\mathrm{C}_{2}-\mathrm{CH}_{3}\right)$; 
$3.00\left(\mathrm{~s}, 3 \mathrm{H}, \mathrm{C} 7-\mathrm{CH}_{3}\right) ; 3.36(\mathrm{t}, 2 \mathrm{H}$, $\left.\mathrm{CH}_{2}-\mathrm{CH}_{2}-\mathrm{CH}_{3}\right) ; 4.95$ (q, $2 \mathrm{H}, \mathrm{CH}_{2}-$ $\left.\mathrm{CH}_{3}\right) ; 6.23$ (s, $2 \mathrm{H}, \mathrm{NH}_{2}$, exchanged by $\left.\mathrm{D}_{2} \mathrm{O}\right) ; 7.86(\mathrm{~d}, 1 \mathrm{H}, \mathrm{C} 5-\mathrm{H}) ; 9.13(\mathrm{~s}$, 1H, C2-H); 9.30 (d, 1H, C6-H).

FAB-MS, $m / z, \%: 345.19[\mathrm{M}+1]^{+}$, $(100 \%), \quad 330.16(19.8 \%), \quad 214.19$

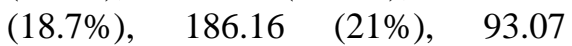
(26.2\%).

\section{3-[4-Amino-5-allylthio-4H-(1,2,4)-} triazol-3-yl]-1-ethyl-7-methyl-1 $H$ $[1,8]$ naphthyridin-4-one (6d)

IR, $v^{-} \mathrm{cm}^{-1}$ (KBr): 3380 and 3260 $\left(\mathrm{NH}_{2}\right), 3055$ (Ar. CH), 2950 (aliph. $\mathrm{CH}), 1620(\mathrm{C}=\mathrm{O}), 1569(\mathrm{NH}, \mathrm{C}=\mathrm{N})$.

${ }^{1} \mathrm{H}-\mathrm{NMR}\left(\mathrm{CDCl}_{3}, \delta \mathrm{ppm}\right): 1.68(\mathrm{t}$, $\left.3 \mathrm{H}, \mathrm{CH}_{2}-\mathrm{CH}_{3}\right) ; 2.95$ (s, 3H, C7- $\mathrm{CH}_{3}$ ); $4.16\left(\mathrm{~d}, 2 \mathrm{H}, \mathrm{s}-\mathrm{CH}_{2}-\mathrm{CH}=\right) ; 5.00(\mathrm{q}$, $\left.2 \mathrm{H}, \mathrm{CH}_{2}-\mathrm{CH}_{3}\right) ; 5.56-5.86(\mathrm{dd}, 2 \mathrm{H}$, $\mathrm{CH}=\mathrm{CH}_{2}$ ); 6.26 (br. s, $2 \mathrm{H}, \mathrm{NH}_{2}$, exchanged by $\left.\mathrm{D}_{2} \mathrm{O}\right)$; 6.95-6.05 (m, $\left.1 \mathrm{H}, \mathrm{CH}_{2}-\mathrm{CH}=\right) ; 7.90(\mathrm{~d}, 1 \mathrm{H}, \mathrm{C} 5-\mathrm{H})$; $9.20(\mathrm{~s}, 1 \mathrm{H}, \mathrm{C} 2-\mathrm{H})$; 9.36(d, 1H, C6$\mathrm{H})$.

EI-MS, $m / z, \%: 342\left(\mathrm{M}^{+}, 53.1 \%\right)$, $326\left(\mathrm{M}^{+}-\mathrm{NH}_{2}, 75.7 \%\right), 214(100 \%)$, $186(81 \%)$.

\section{3-[4-Amino-5-benzylthio-4H-(1,2,} 4)triazol-3-yl]-1-ethyl-7-methyl-1H $[1,8]$ naphthyridin-4-one (6e)

IR, $v^{-} \mathrm{cm}^{-1}$ (KBr): 3400 and 3265 $\left(\mathrm{NH}_{2}\right), 3050$ (Ar. CH), 2950 (aliph. $\mathrm{CH}), 1617(\mathrm{C}=\mathrm{O}), 1558(\mathrm{NH}, \mathrm{C}=\mathrm{N})$, 782 and 708 (monosubstituted benzene).

${ }^{1} \mathrm{H}-\mathrm{NMR}\left(\mathrm{CDCl}_{3}, \delta \mathrm{ppm}\right): 1.76(\mathrm{t}$, $\left.3 \mathrm{H}, \quad \mathrm{CH}_{2}-\mathrm{C}_{3}\right) ; 3.00 \quad(\mathrm{~s}, 3 \mathrm{H}, \quad \mathrm{C} 7-$ $\left.\mathrm{CH}_{3}\right) ; 5.30-4.63\left(\mathrm{~m}, 4 \mathrm{H}, \mathrm{CH}_{2}-\mathrm{CH}_{3}\right.$ and $\mathrm{C}_{2}$-ph); 6.16 (brs, $2 \mathrm{H}, \mathrm{NH}_{2}$, exchanged by $\left.\mathrm{D}_{2} \mathrm{O}\right)$; 8.33-7.70 (m,
6H, C6H5 and $\mathrm{C} 5-\mathrm{H}) ; 9.22(\mathrm{~s}, 1 \mathrm{H}$, $\mathrm{C} 2-\mathrm{H})$; 9.36(d, 1H, C6-H).

EI-MS, $m / z, \%: 392\left(\mathrm{M}^{+}, 15.3 \%\right)$, $376\left(\mathrm{M}^{+}-\mathrm{NH}_{2}, 36.4 \%\right), 214(40.7 \%)$, $186(96.6 \%), 91$ (100\%).

\section{Synthesis of 3-\{[4-(un)substituted} benzylidene-amino]-5-benzylthio$4 H-(1,2,4) \quad$ triazol-3-yl]\}-1-ethyl-7methyl-1H-[1,8]naphthyridin-4-one derivatives $(\mathbf{7 a}, \mathbf{b})$

Benzyl chloride (0.15 g, 0.0012 $\mathrm{mol})$ in absolute ethanol $(30 \mathrm{~mL})$ was added to the appropriate Schiff's bases $(\mathbf{5 a}, \mathbf{e})(0.001 \mathrm{~mol})$ dissolved in a least amount of $10 \% \mathrm{NaOH}$ and refluxed for 15 hrs. The solid obtained was filtered, washed with water and crystallized from methanol as white solids.

3-\{[4-Benzylidene-amino]-5-benzylthio-4H-(1,2,4)triazol-3-yl]\}-1ethyl-7-methyl-1H-[1,8]naphthyridin-4-one (7a): Yield; (69\%), m.p. $208-210^{\circ} \mathrm{C}$.

IR, $v^{-} \mathrm{cm}^{-1}(\mathrm{KBr}): 3025$ (Ar. CH), 2940 (aliph. CH), $1616(\mathrm{C}=\mathrm{O}), 1582$ $(\mathrm{C}=\mathrm{C}, \quad \mathrm{C}=\mathrm{N}), \quad 746, \quad 690 \quad$ (monosubstituted benzene).

${ }^{1} \mathrm{H}-\mathrm{NMR}, \delta$ ppm $\left(\right.$ DMSO- $\left._{6}\right): 1.53$ (t, $\left.3 \mathrm{H}, \mathrm{CH}_{2}-\mathrm{CH}_{3}\right) ; 2.86$ (s, 3H, C7$\left.\mathrm{CH}_{3}\right) ; 5.25-4.60\left(\mathrm{~m}, 4 \mathrm{H}, \mathrm{CH}_{2}-\mathrm{CH}_{3}\right.$ and $\left.\mathrm{C}_{2}-\mathrm{ph}\right) ; 8.50-7.70(\mathrm{~m}, 11 \mathrm{H}$, $2 \mathrm{C}_{6} \underline{\mathrm{H}}_{\underline{5}}$ and $\left.\mathrm{N}=\mathrm{CH}\right) ; 9.03(\mathrm{~d}, 1 \mathrm{H}, \mathrm{C} 5-$ $\underline{\mathrm{H}}$; 9.32 (s, $2 \mathrm{H}, \mathrm{C} 2-\underline{\mathrm{H}}$ and $\mathrm{C} 6-\underline{\mathrm{H}})$.

EI-MS, $m / z(\%): 480\left[\mathrm{M}^{+}, 28.4 \%\right]$, 186 [100\%], 214 [93.9\%], 91 [70.1\%]. Anal. Calcd. for $\mathrm{C}_{27} \mathrm{H}_{24} \mathrm{~N}_{6} \mathrm{OS} . \mathrm{H}_{2} \mathrm{O}$ (M.W. 498.18): $\% \mathrm{C} 65.03, \% \mathrm{H} 4.85, \% \mathrm{~N} \mathrm{16.86, \% \textrm {S }}$ 6.41. Found: $\% \mathrm{C} 65.11, \% \mathrm{H} \mathrm{5.42,}$ $\% \mathrm{~N} \mathrm{16.98, \% S} \mathrm{7.08.}$ 
3-\{[4-(p-Methoxy)benzylideneamino]-5-benzylthio- $4 H-(1,2,4)-$ triazol-3-yl]\}-1-ethyl-7-methyl-1 H[1,8]naphthyridin-4-one (7b): Yield; (65\%), m.p. $229-231^{\circ} \mathrm{C}$.

IR, $v \mathrm{~cm}^{-1}(\mathrm{KBr}): 3030$ (Ar. CH), 2950 (aliph. $\mathrm{CH}$ ), $1616(\mathrm{C}=\mathrm{O}), 1540$ $(\mathrm{C}=\mathrm{C}, \mathrm{C}=\mathrm{N}), 1250\left(\mathrm{SP}^{2} \mathrm{C}-\mathrm{O}\right), 1168$, 1027 ( SP $^{3}$ C-O), 820 (p-disubstituted benzene), 787, 691 (monosubstituted benzene).

${ }^{1} \mathrm{H}-\mathrm{NMR}, \delta \mathrm{ppm}$ (DMSO-d $\mathrm{d}_{6}$ ): 1.55 (t, 3H, $\left.\mathrm{CH}_{2}-\mathrm{CH}_{3}\right) ; 2.92$ (s, 3H, C7$\left.\mathrm{CH}_{3}\right) ; 4.16$ (s, $\left.3 \mathrm{H}, \mathrm{O}-\mathrm{C}_{3}\right) ; 5.30-4.56$ (m, $4 \mathrm{H}, \mathrm{CH}_{2}-\mathrm{CH}_{3}$ and $\mathrm{CH}_{2}-\mathrm{ph}$ ); 8.55$7.45 \quad\left(\mathrm{~m}, \quad 10 \mathrm{H}, \quad \mathrm{C}_{6} \underline{\mathrm{H}}_{5}, \quad \mathrm{C}_{6} \underline{\mathrm{H}}_{4}\right.$ and $\mathrm{N}=\mathrm{C} \underline{\mathrm{H}}$ ); 9.56-8.96 (m, 3H, C2-프, C5$\mathrm{H}$ and $\mathrm{C} 6-\mathrm{H})$.

Anal. Calcd. for $\mathrm{C}_{28} \mathrm{H}_{26} \mathrm{~N}_{6} \mathrm{O}_{2} \mathrm{~S} .1 / 2$ $\mathrm{H}_{2} \mathrm{O}$ (M.W. 519.18): \%C 64.71, \%H $5.05, \% \mathrm{~N} 16.18, \% \mathrm{~S} 6.15$. Found: \%C $64.21, \% \mathrm{H} 5.61, \% \mathrm{~N} 16.51, \% \mathrm{~S} 6.86$.

\section{Antimicrobial screening \\ Antibacterial activity}

The antibacterial activity of all target compounds (4, 5a-f, 6a-e and $7 \mathbf{a}, \mathbf{b})$ was investigated in-vitro at the department of microbiology and immunology, faculty of medicine, Assiut University. The title compounds were tested against methicillin resistant Staphylococcus aureus (MRSA), Bacillus cereus, Escherichia coli, and Klebsiella pneumoniae (clinical isolates obtained from Infection Control Unit, Assiut University Hospital, Faculty of Medicine, Assiut University) using agar cup diffusion method ${ }^{19 \& 20}$ for susceptibility screening, and two-fold dilution method ${ }^{20}$ for MIC determination. Ampicillin was used as a reference antibiotic, and DMSO was used as a solvent control.

\section{Agar cup diffusion method}

$38 \mathrm{Gm}$ of Mueller-Hinton agar medium (MH) (Hi-Media, M 001) were added to $1 \mathrm{~L}$ of distilled water, heated to boiling to dissolve the ingredients completely, and sterilized by autoclaving at $121^{\circ} \mathrm{C}$ for 30 mins. High density inocula were made by diluting 3-5 well isolated colonies grown overnight on selective media in $5 \mathrm{~mL}$ of distilled water to prepare a suspension equivalent in density to 0.5 McFarland Barium Sulfate standard unit with average turbidity $10^{8} \mathrm{CFU} \mathrm{mL}^{20}$. The sterile petri dishes were seeded with $100 \mathrm{~L}$ of the microorganism; a specified amount of the molten $\mathrm{MH}$ agar medium $\left(45-50^{\circ} \mathrm{C}\right)$ was poured into the seeded Petri dishes to give a depth of 3-4 $\mathrm{mm}$ and allowed to solidify. Cylindrical plugs were removed from the agar using sterile cork borer. 100

$\mathrm{L}$ of the tested compounds (20 $\mathrm{mg} / \mathrm{mL}$ in DMSO), the blank solvent, and ampicillin sodium $(20 \mathrm{mg} / \mathrm{mL}$ in DMSO) were added to the wells in triplicate. The seeded plates were incubated at $37^{\circ} \mathrm{C}$ for $24 \mathrm{hrs}$ then the average diameters of the inhibition zones were measured in millimeters (Table III).

\section{Minimum inhibitory concentration}

The MIC values were determined using two fold-dilution method ${ }^{21}$ for compounds having moderate to strong antibacterial activity. The squares of inhibition zone diameters were plotted against log concentrations of the tested compounds, extrapolation 
Table III: Antibacterial Activity of The Tested Compounds (expressed as the inhibition zone diameter and as $\mathrm{MIC} \mu \mathrm{M} / \mathrm{mL}$ ).

\begin{tabular}{|c|c|c|c|c|c|c|c|c|}
\hline \multirow[b]{2}{*}{$\begin{array}{l}\text { Compd. } \\
\text { No. }\end{array}$} & \multicolumn{2}{|c|}{ MRSA } & \multicolumn{2}{|c|}{ Bacillus cereus } & \multicolumn{2}{|c|}{ E. coli } & \multicolumn{2}{|c|}{$\begin{array}{c}\text { Klebsiella } \\
\text { Pneumoniae }\end{array}$} \\
\hline & $\begin{array}{c}\text { Inhibition } \\
\text { zone } \\
(\mathrm{mm})\end{array}$ & $\begin{array}{c}\mathrm{MIC} \\
\mu \mathrm{M} / \mathrm{ml}\end{array}$ & $\begin{array}{c}\text { Inhibition } \\
\text { zone } \\
(\mathrm{mm})\end{array}$ & $\begin{array}{c}\mathrm{MIC} \\
\mu \mathrm{M} / \mathrm{ml}\end{array}$ & $\begin{array}{c}\text { Inhibition } \\
\text { zone } \\
(\mathrm{mm})\end{array}$ & $\begin{array}{c}\mathrm{MIC} \\
\mu \mathrm{M} / \mathrm{ml}\end{array}$ & $\begin{array}{c}\text { Inhibition } \\
\text { zone } \\
(\mathrm{mm})\end{array}$ & $\begin{array}{c}\mathrm{MIC} \\
\mu \mathrm{M} / \mathrm{ml}\end{array}$ \\
\hline 4 & 15 & & 13 & & 11 & & 17 & \\
\hline $5 \mathbf{a}$ & 21 & 75 & 23 & 69.1 & 24 & 65 & 26 & 35 \\
\hline $5 \mathbf{b}$ & 21 & 70 & 23 & 61 & 25 & 50 & 24 & 57 \\
\hline $5 c$ & 20 & 70 & 22 & 60 & 26 & 35 & 27 & 30 \\
\hline $5 d$ & 16 & & 15 & & 14 & & 12 & \\
\hline $5 e$ & 21 & & -ve & & -ve & & -ve & \\
\hline $5 f$ & -ve & & -ve & & -ve & & -ve & \\
\hline $6 \mathbf{a}$ & 9 & & -ve & & $-\mathrm{ve}$ & & -ve & \\
\hline $\mathbf{6 b}$ & 28 & 25 & 22 & 60 & 27 & 30 & 27 & 30 \\
\hline $6 c$ & 18 & & 15 & & 13 & & 9 & \\
\hline 6d & 20 & 69.1 & 22 & 72 & 21 & 75 & 23 & 70 \\
\hline $6 e$ & 20 & 70 & 23 & 69.1 & 22 & 71 & 25 & 40 \\
\hline $7 \mathbf{a}$ & -ve & & 6 & & 7 & & -ve & \\
\hline $7 \mathbf{b}$ & 10 & & $-\mathrm{ve}$ & & 10 & & 7 & \\
\hline Ampicillin & 20 & 69 & 22 & 60 & 20 & 70 & 23 & 50 \\
\hline $\begin{array}{c}\text { Nalidixic } \\
\text { acid }\end{array}$ & 27 & 30 & 20 & 67 & 28 & 25 & 30 & 20 \\
\hline
\end{tabular}

of the resulting straight line to intersect with log concentration scale in the curve corresponded to $\log$ MIC, and MIC was obtained as antilog ${ }^{22}$ (Table III).

\section{Antifungal activity}

\section{Organisms and culture conditions}

The synthesized compounds (4, 5a-f, 6a-e, 7a and $7 \mathbf{b}$ ) were tested for their antifungal activity in-vitro, in comparison with clotrimazole as a reference drug and nalidixic acid as a reference starting material, using the standard agar cup diffusion method ${ }^{22}$ at the Assiut University Mycological Center (AUMC), Faculty of Science, Assiut University.
Seven pathogenic, phytopathogenic or food poisoning fungal species were used in the present study: Trichophyton rubrum (AUMC 1804), Candida albicans (AUMC 5109), Fusarium oxysporium (AUMC 3224), Penicillium chrysogenum (AUMC 277), Aspergillus terreus (AUMC 2726), Drechslera spicifier (AUMC 5110) and Micosporum gypseum (AUMC 5095).

\section{Materials and method ${ }^{23}$}

Spore suspension in sterile malt extract broth media was prepared from 2-5 days old culture of the test fungi growing on malt extract agar as in case of Aspergillus terreus and Penicillium chrysogenium, sabouraud 
agar as in case of Candida albicans, Drechslera spicifier, Micosporum gypseum and Trichophyton rubrum, or on potato dextrose agar as in case of Fusarium oxysporium. The final spore concentration was $5 \times 10^{4}$ spores/ ml. About $15 \mathrm{ml}$ of growth medium was introduced on sterilized Petri dishes of $9 \mathrm{~cm}$ diameter and inoculated with $1 \mathrm{ml}$ spore suspension. Plates were shaken gently to homogenize the inoculum. Antifungal activity of the tested compounds was performed by the standard agar cup diffusion method as follow: Cylindrical plugs were removed from the agar using a sterile cork bore. $100 \mu \mathrm{l}$ of the tested compounds (100 $\mu \mathrm{mol} / \mathrm{ml}$ in DMSO) and the blank solvent were added to each well in triplicate. The seeded plates were incubated at $28 \pm 2^{\circ} \mathrm{C}$ for 1-7 days according to fungi used. After the specified time for incubation the average diameter of inhibition zones was measured in millimeters (Table IV). Solution of nalidixic acid and clotrimazole (100 $\mu \mathrm{mol} / \mathrm{ml}$ in DMSO) were used as reference starting compound and as standard antifungal agent.

Table IV: Antifungal activity of the tested compounds (expressed as the diameter of the inhibition zone* in $\mathrm{mm}$ ).

\begin{tabular}{|c|c|c|c|c|c|c|c|}
\hline $\begin{array}{l}\text { Fungi } \\
\text { Comp. } \\
\text { No. }\end{array}$ & 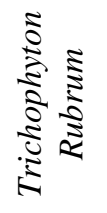 & 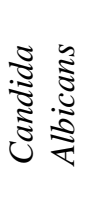 & 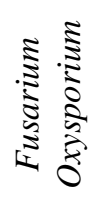 & 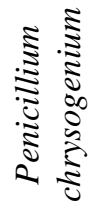 & 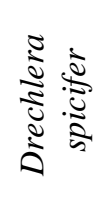 & 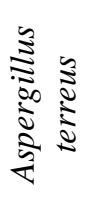 & 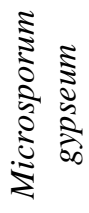 \\
\hline 4 & 0 & 0 & 0 & 0 & 22 & 0 & 0 \\
\hline $5 \mathbf{a}$ & 0 & 0 & 0 & 0 & 0 & 0 & 22 \\
\hline $5 \mathbf{b}$ & 0 & 0 & 0 & 0 & 0 & 0 & 0 \\
\hline $5 \mathrm{c}$ & 0 & 0 & 0 & 0 & 0 & 0 & 0 \\
\hline $5 d$ & 20 p.i & 0 & 0 & 0 & 0 & 0 & 0 \\
\hline $5 e$ & 0 & 0 & 0 & 0 & 0 & 0 & 22 \\
\hline $5 f$ & 0 & 0 & 0 & 0 & 0 & 0 & 0 \\
\hline $6 a$ & 0 & 0 & 0 & 0 & 0 & 0 & 0 \\
\hline $6 \mathbf{b}$ & 0 & 0 & 0 & 0 & 0 & 0 & 20 \\
\hline $6 c$ & 0 & 0 & 0 & 0 & 0 & 0 & 0 \\
\hline 6d & 0 & 0 & 0 & 0 & 0 & 0 & 0 \\
\hline $6 e$ & 0 & 0 & 0 & 0 & 0 & 0 & 0 \\
\hline $7 \mathbf{a}$ & 0 & 0 & 0 & 0 & 0 & 0 & 0 \\
\hline $7 \mathbf{b}$ & 0 & 0 & 0 & 0 & 0 & 0 & 24 \\
\hline Nalidixic acid & 18 p.i & 0 & 0 & 0 & 12 p.i & 0 & 16 \\
\hline Clotrimazole & 58 & 35 & 25 & 55 & 30 & 27 & 58 \\
\hline
\end{tabular}

*Average of three determinations p.i partial inhibition 


\section{RESULTS AND DISCUSSION}

\section{Chemistry}

Nalidixic acid hydrazide $\mathbf{3}$ was prepared in analogy to the reported procedure $^{15-16}$ by refluxing the corresponding methyl ester $\mathbf{2}$ with hydrazine hydrate in methanol (Scheme 1). The structure of compounds $\mathbf{2}$ and $\mathbf{3}$ was confirmed by matching its physical data with the reported.

The key intermediate in this work, compound 4; 3-[4-amino-5-thioxo4,5-dihydro-1H-1,2,4-triazol-3-yl]-1ethyl-7-methyl-1H-[1,8]naphthyridin4-one; was prepared by the condensation of the hydrazide 3 with $\mathrm{CS}_{2}$ and ethanolic $\mathrm{KOH}$ to afford the corresponding potassium dithiocarbazate salt. The salt was cyclized, at refluxing temperature, with hydrazine hydrate $97 \%$ to furnish the triazole $\mathbf{4}$, which is a new compound. The structure of compound $\mathbf{4}$ was confirmed by IR, ${ }^{1} \mathrm{H}-\mathrm{NMR}$ spectra as well as elemental analysis. IR spectrum of compound $\mathbf{4}$ showed a broad band at $3455 \mathrm{~cm}^{-1}\left(\mathrm{NH}_{2}, \mathrm{NH}\right)$ stretching, and a medium band at $1251 \mathrm{~cm}^{-1}(\mathrm{C}=\mathrm{S})$ stretching which revealed that compound $\mathbf{4}$ present in the thione form in the solid state ${ }^{24}$. Also, the absence of absorption at about 2600-2550 region cited for SH group have proved that the compound was in the thionic form.

The Schiff's bases (compounds 5a-f) were prepared by reaction of the triazole 4 with one equivalent of (un)substituted benzaldehydes in ethanol and few drops of concentrated $\mathrm{HCl}$ acid as catalysis (Scheme 1).

Comparative study of the ${ }^{1} \mathrm{H}$ NMR spectra of these Schiff's bases with compound 4 easily revealed the disappearance of the broad singlet at $\delta 6.13 \mathrm{ppm}$ corresponding to $\mathrm{NH}_{2}$ group present in compound $\mathbf{4}$ and appearance of $\mathrm{N}=\mathrm{CH}$ signals at $10.56-11.20 \mathrm{ppm}$ in addition to the marked change in the aromatic protons number and splitting pattern, which proofs the introduced aromatic moiety. A representative FAB-MS spectrum was done for compound $\mathbf{5 e}$ (M.F. $=\quad \mathrm{C}_{21} \mathrm{H}_{20} \mathrm{~N}_{6} \mathrm{O}_{2} \mathrm{~S}, \quad$ M.Wt. $=$ 420.14) showed the adduct ion peak $\left[\mathrm{M}^{+}+1\right]$ at $(\mathrm{m} / \mathrm{z}$ 421.18; intensity $2.2 \%$ ), as well as prominent peaks at $\mathrm{m} / z$ : 287.93 (100\%), 214.01 (36.8\%), $185.95(46.4 \%), 92.94(49.4 \%)$ and $78.95(86.2 \%)$.

Reaction of compound 4 with equimolar amount of the appropriate alkyl or aralkyl halide in presence of $\mathrm{NaOH}$ in ethanol at refluxing temperature, afforded the S-alkyl derivatives (compounds 6a-e) Scheme 1. It was reported that $\mathrm{S}$ alkylation supercsedes the Nalkylation due to of the difference in nucelophilicity between the sulfur and nitrogen atoms ${ }^{25 \& 26}$. The IR spectra of compounds 6a-e showed a broad band at $3400-3260 \mathrm{~cm}^{-1}$ (stretching) and at $1568 \mathrm{~cm}^{-1}$ (bending) of the $\mathrm{NH}_{2}$. The ${ }^{1} \mathrm{H}-\mathrm{NMR}$ spectra showed the presence of a broad singlet at $\delta$ 6.16-6.40 ppm corresponding to $\mathrm{NH}_{2}$ protons and the absence of the broad singlet at $\delta$ 12.5- $15.37 \quad(\mathrm{NH})$. Moreover the S-alkyl groups give a 


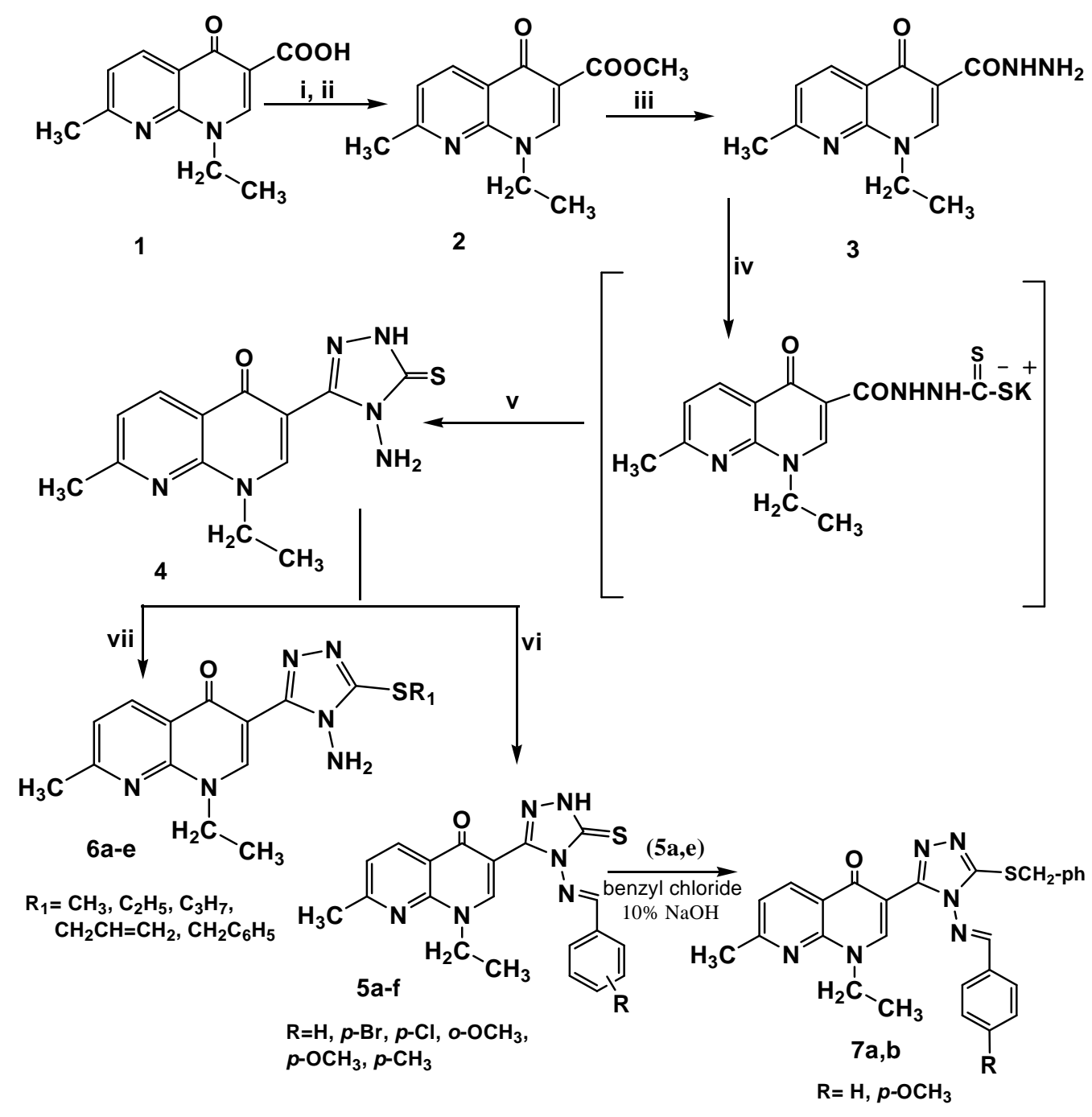

Scheme 1

Reagents and conditions: (i) $\mathrm{ClCOOCH}_{2} \mathrm{CH}_{3}$, Triethylamine, $\mathrm{CHCl}_{3}, 5-10^{\circ} \mathrm{C}$; (ii) $\mathrm{CH}_{3} \mathrm{OH}$; (iii) $\mathrm{N}_{2} \mathrm{H}_{4} \cdot \mathrm{H}_{2} \mathrm{O} 80 \%, \mathrm{CH}_{3} \mathrm{OH}$, reflux, 6 hrs; (iv) $\mathrm{CS}_{2}, \mathrm{KOH}, \mathrm{EtOH}$, $24^{\circ} \mathrm{C}, 20 \mathrm{hrs}$; (v) $\mathrm{N}_{2} \mathrm{H}_{4} \cdot \mathrm{H}_{2} \mathrm{O} 97 \%$, reflux, $2 \mathrm{hrs}$; (vi) appropriate (un)substituted benzaldehydes, EtOH, reflux, 20 hrs; (vii) appropriate alkyl or aralkyl halide, $10 \% \mathrm{NaOH}, \mathrm{EtOH}$, reflux, $15 \mathrm{hrs}$. 
pattern in the ${ }^{1} \mathrm{H}-\mathrm{NMR}$ spectra in accordance with the expected structures of such compounds.

A representative EI-MS spectrum was done for compound $\mathbf{6 d}$ showed the molecular ion peak $\mathrm{M}^{+}$at $(\mathrm{m} / \mathrm{z}$ 342 ; intensity $53.1 \%$ ), corresponding to the molecular weight of the compound and prominent peaks at $(\mathrm{m} / \mathrm{z} 326 ; 75.7 \%),(\mathrm{m} / \mathrm{z} 214 ; 100 \%)$ and $(\mathrm{m} / \mathrm{z}, 186 ; 81 \%)$.

Moreover, treatment of the Schiff's bases 5a,e with benzyl chloride afforded S-benzyl derivatives (7a,b) of the two Schiff's bases. The structures of the formed Schiff's bases were verified by IR, ${ }^{1} \mathrm{H}-\mathrm{NMR}$, EI or FAB-MS and elemental analyses. IR and ${ }^{1} \mathrm{H}-\mathrm{NMR}$ showed neither NH nor $\mathrm{NH}_{2}$ and appearance of $\mathrm{N}=\mathrm{CH}$ in addition to aromatic protons (exp. Part).

\section{Antimicrobial activities Antibacterial activity}

The newly synthesized compounds 4, 5a-f, 6a-e and 7a,b were tested for their in-vitro antibacterial activity against methicillin resistant Staphylococcus aureus (MRSA) and Bacillus cereus as representatives of Gram-positive strains and Escherichia coli and Klebsiella pneumoniae as representatives of Gram-negative ones using nalidixic acid and ampicillin as reference drugs. The results revealed that most of the newly synthesized compounds exhibited promising antibacterial activity against all the test organisms (Table III). The synthesized Schiff's bases, compounds 5a-c and the Salkyl derivatives compounds $\mathbf{6 b}$, d, and 6e were the most potent against all strains. Their antibacterial potency was superior to that of ampicillin against all the tested strains and higher than that of nalidixic acid against Bacillus cereus. They showed comparable antibacterial activity to nalidixic acid against methicillin resistant Staphylococcus aureus (MRSA), Escherichia coli and Klebsiella pneumoniae.

In general, screening results revealed that replacement of the carboxylic acid group at position 3- of nalidixic acid with the triazole group (compound 4), causes marked decrease in the antibacterial activity. Among the Schiff's bases, (compounds 5d,e), having methoxy group in the benzylidene moiety, did not exhibit enhanced activity compared to the other bases having no substituent or substituted by halogen. Substitution by methyl group led to loss of antibacterial activity, compound $\mathbf{5 f}$.

Also, it has been observed that alkylation of compound $\mathbf{4}$ with methyl group, (compound 6a), decreases the activity and alkylation with n- propyl (compound 6c), slightly increases it, while alkylation with ethyl, allyl or benzyl group showed higher activity than ampicillin, with the most active one is (compound $\mathbf{6 b}$ ). In conclusion, the substitution of the carboxylic group of nalidixic acid by unsubstituted 1,2,4-triazole moiety or S-alkyl derivatives of Schiff's bases decreases the antibacterial activity. In 
general anticipation of SAR can not be attained.

\section{Antifungal activity}

Unfortunately, results of the antifungal activity (Table IV) revealed that all the test compounds and nalidixic acid were inactive against $C$. albicans, $F$. oxysporium, $P$. chrysogenum and $A$. terreus. Also they were inactive against $D$. spicifier except nalidixic acid which showed partial inhibition $40 \%$ relative to clotrimazole. The test compounds were inactive against $T$. rubrum except compounds 5d which showed $34 \%$ inhibition and nalidixic acid showed $31 \%$ inhibition relative to the standard. It was also noticed that many compounds showed moderate activity against $M$. gypseum (compounds $\mathbf{6 b}, \mathbf{5 a}, \mathbf{5 d}, \mathbf{5 e}$ and $\mathbf{7 b}$ showed 34,38,34,38,41\% inhibition respectively), while nalidixic acid showed weak inhibition (27.6\%).

From the above results, it was clear that conversion of compound 4 to Schiff's bases (5a, 5d and $\mathbf{5 e}$ ) and some S-alkylation (6b and $7 \mathbf{b})$ is critical for the antifungal activity, with compound $\mathbf{7 b}$ was the most active one.

Also, screening tests are quiet consistent with the reported literature that nalidixic acid has weak or no antifungal activity ${ }^{27 \& 28}$.

\section{REFERENCES}

1- G. Gaurav and S.G. Kini, Eur. J. Med. Chem., 41, 256 (2006).
2- R. Albrecht, Prog. Drug Res., 21, 9 (1977).

3- M. I. El-Zahar, M. M. Kamel and E. M. Mohei El-Deen, Egypt. J. Chem., 45, 323 (2002).

4- R. A. Mekheimer, A. M. Abdel Hameed and K. U. Sadek, Arkivoc, (xiii), 269 (2007).

5- A. S. Noravyan, E. G. Paronikyan and S. A. Vartanyan, J. Pharm. Chem., 19, 439 (1985).

6- G. Gaurav and S. G. Kini, Indian J. Heterocycl. Chem., 12, 289 (2003).

7- A. F. Youssef, F.A. Omar, H. A. Elsherief and G. E. A. AbuoRahma, Bull. Pharm. Sci., Assiut University, 21, 15 (1998).

8- A. M. Sugar, X. P. Liu and R. J. Chen, Antimicrob. Agents Chemother., 41, 2518 (1997).

9- Y. A. Al-Soud and N. A. AlMasoudi, J. Braz. Chem. Soc., 14, 790 (2003).

10- Y. He, J. Yang, B. Wu, L. Riesen and E. Swayze, Bioorg. Med. Chem. Lett., 14, 1217 (2004).

11- K. C. Ragenovic, V. Dimova, V. Kakurinov, D. G. Molnar and A. Buzrouska, Molecules, 6, 815 (2001).

12- R. M. Shaker and A. A. Aly, Phosphorus, Sulfur, and Silicon, 181, 2577 (2006).

13- N. F. Eweiss, A. A. Bahajaj and E. A. Elsherbini, J. Heterocycl. Chem., 23, 1451 (1986).

14- G. Grossi, M. Di Braccio, G. Roma, V. Ballabeni, M. Tognolini and E. Barocelli, Eur. J. Med. Chem., 40, 155 (2005). 
15- F. A. Omar, Alex. J. Pharm. Sci., 8, 65 (1994).

16- G. A. A. Abuo-Rahma, Master Thesis, Assiut University, Assiut, Egypt (1998).

17- M. G. Mamolo, V. Falagiani, D. Zampieri, L. Vio, and E. Banfi, Farmaco, 56, 587 (2001).

18- A. Foroumadi, S. Emami, A. Hassanzadeh, M. Rajaee, K. Sokhanvar, M. H. Moshafi and A. Shafiee, Bioorg. Med. Chem. Lett., 15, 4488 (2005).

19- A. M. Clark, F. S. El-Feraly and W. Li, J. Pharm. Sci., 70, 951 (1981).

20- A. Felten, B. Grandly, P. H. Lagrange and I. Casin, J. Clin. Microbiol., 40, 2766 (2002).

21- J. G. Collee, J. P. Duguid, A. G. Fraser and B. P. Marmion, "Practical Medical Microbiolgy", $3^{\text {rd }}$ Ed. (Churchill Livingstone) London, New York, Vol. 2, 1989, pp. 161-289.

22- W. Hewitt, "Microbiological Assay, An Introduction to Quantitative Principles and Evaluation", (Academic press) New York, London, 1977, pp. 17-69.
23- M. A. El-Sherbeny, M. B. ElAshmawy, A. A. El- Subbagh and F. A. Badria, Eur. J. Med. Chem., 30, 445 (1995).

24- M. Biagini C., F. Bigoli, M. Lanfranchi, E. Leporati, M. Angela P. and C. Foglia, Inoganica Chimica Acta, 235, 37 (1995).

25- D. M. Smith, In "Chemistry of Heterocyclic Compounds", 40, I, Weissberger, A., editor, John Wiely and Sons, New York, 1981, p. 360.

26- N. N. Gülerman, N. H. Doöan, S. Rollas, C. Johansson and C. Celik, Farmaco, 56, 953 (2001).

27- C. A. Michels, J. Blamire, B. Goldfinger and J. Marmur, Antimicrob. Ag. Chemother., 3, 562 (1973).

28- S. Ahmed, M. H. Bhatti, S. Ali and F. Ahmed, Turk. J. Chem., 30, 193 (2006). 